\title{
甲氨蝶呤/层状双金属氢氧化物的粒径调控及缓释性能研究
}

\author{
齐凤林李淑萍*张晓晴 \\ (江苏省生物功能材料重点实验室 南京师范大学化学与材料科学学院 南京 210097)
}

\begin{abstract}
摘要 在乙醇一水混合体系中, 以氨水为沉淀剂共沉淀合成了甲氨蝶呤/层状双金属氢氧化物(MTX/LDH)纳米复合物, 首次采用控制沉淀剂滴加速率的方式来调控其粒径. 利用 X-射线衍射(XRD)、透射电镜(TEM)和红外光谱(FT-IR)等表 征手段, 对其结构及形貌进行了表征. 研究表明: MTX 分子以单层倾斜方式插入 LDH 层间, 随着滴加速率不同, MTX 在层间的倾斜角度发生了变化; 沉淀剂滴加速率对产物的结晶度、粒径和层间排列方式都有影响, 当沉淀剂滴加速率 为 $0.100 \mathrm{~mL} / \mathrm{s}$ 时, 得到的 $\mathrm{MTX} / \mathrm{LDH}$ 纳米复合物的结晶度最高, 粒径最大. 在磷酸缓冲液中考察了不同粒径 MTX/LDH 纳米复合物的缓释性能, 结果表明: 小粒径的 MTX/LDH 纳米复合物的载药量要高于大粒径的; 当 MTX 阴离子在层间 的倾斜角度相似时, 粒径小的粒子释放速率较慢, 缓释效果优于粒径大的. 尤为重要的是我们探索出一条保证复合物 阴离子在层间的倾斜角度相似的情况下, 制备不同粒径纳米复合物的新途径.

关键词＼cjkstart纳米粒子; 层状化合物; 缓释性能; 沉淀剂滴加速率; 甲氨蝶呤
\end{abstract}

\section{Controllable Synthesis and Release Properties of Methotrexatum/Layered Double Hydroxide Compounds}

\author{
Qi, Fenglin Li, Shuping* Zhang, Xiaoqing \\ (Jiangsu Key Laboratory of Biofunctional Materials, College of Chemistry and Materials Science, Nanjing \\ Normal University, Nanjing 210097, China)
}

\begin{abstract}
The methotrexatum/layered double hydroxide (MTX/LDH) compounds with different particle sizes were prepared by controlling the dropping speed of precipitator, using the coprecipitation method in alcohol-water solvent and $\mathrm{NH}_{3} \cdot \mathrm{H}_{2} \mathrm{O}$ as precipitator. The influence of the dropping speed of precipitator on the structure and morphologies of MTX/LDHs was systematically characterized by X-ray diffraction (XRD), transmission electron micrograph (TEM) and fourier transform infrared spectroscopy (FT-IR). The results indicated that MTX anions were intercalated into the LDH interlayers with a declined monolayer and the angle of inclination changed with the variation of the dropping speed of precipitator. Furthermore, the dropping speed of precipitator affected the crystallinity and particle size as well as arrangement way of MTX in LDH interlayers, when the dropping speed is $0.100 \mathrm{~mL} / \mathrm{s}$, the products have the highest crystallinity and the maximum diameter. All the MTX/LDHs particles exhibited hexagonal platelet morphology with round corner and good monodispersity. At last, the release properties of MTX/LDHs with different particle sizes were studied in phosphate buffer. The results indicated that the smaller nanoparticles have higher drug-loading capacity than those of the larger ones and when the angles of inclination keep almost constant, the smaller nanoparticles have the longer release time than those of the larger ones. In addition, four kinetic models (first-order equation, Higuchi equation, Bhaskar equation and Ritger-Peppas equation) were used to study the release kinetics of MTX from the LDH interlayers, and it was found that the mechanism for drug release can be well described by the Ritger-Peppas equation, revealing that the release mechanism of MTX/LDH belongs to drug diffusion and Fickian diffusion is the rate limiting step. First of all, we have explored a new way to synthesize LDH compounds with different particle size in the guarantee of the angles of inclination of anions in interlayer keeping similar.

Keywords nanoparticles; layered compound; release property; dropping speed of precipitator; methotrexatum
\end{abstract}

\section{1 引言}

新型药物载体的研发已成为当今药物传递领域的 重要研究方向之一, 理想的药物载体应该具有良好的生 物相容性、可降解性、稳定性以及极低的毒性. 层状双 金属氢氧化物(layered double hydroxide compounds, 简 称 LDH)作为一种新型的生物药物输送载体, 因其具有 独到的优势，如安全性高、稳定性好、生物利用度高和 良好的可生物降解性等特点而成为极具有应用前景的 新型纳米药物载体 ${ }^{[1]}$.

\footnotetext{
*E-mail: lishuping@njnu.edu.cn

Received March 25, 2012; published August 23, 2012.

Supporting information for this article is available free of charge via the Internet at http://sioc-journal.cn.

Project supported by the National Natural Science Foundation of China (No. 21073093), Research Fund for the Doctoral Program of Higher Education of China (No. 20103207120006) and the Priority Academic Program Development of Jiangsu Higher Education Institutions.

项目受国家自然科学基金(No. 21073093)、高等学校博士点专项科研基金(No. 20103207120006)和江苏高校优势学科建设工程资助.
} 
层状双金属氢氧化物, 也称为类水滑石 (hydrotalcite like compounds, 简写为 HTlcs), 是一类由 带正电荷的金属氢氧化物层和层间充斥的可交换阴离 子所构成的层柱状化合物, 化学通式可表示为: $\left[\mathrm{M}_{1-x}^{2+} \mathrm{M}_{x}^{3+}(\mathrm{OH})_{2}\right]^{x+}\left[\mathrm{A}^{n-}\right]_{x / n} \bullet m \mathrm{H}_{2} \mathrm{O}$, 其中 $\mathrm{M}^{2+}$ 表示 +2 价 金属离子, $\mathrm{M}^{3+}$ 表示 +3 价金属离子, $\mathrm{A}^{n-}$ 表示层间阴离 子, $x$ 为每摩尔 $\mathrm{LDHs}$ 中 $\mathrm{M}^{3+}$ 的摩尔数; $m$ 是每摩尔 $\mathrm{LDHs}$ 中结晶水的摩尔数 ${ }^{[2 \sim 4]}$. 由于 $\mathrm{LDH}$ 具有层间阴离子的可 交换性 ${ }^{[5]}$ 和层间空间的可调性，因此，可以将某些无机、 有机、配合物阴离子以及一些生物药物分子等插入其层 间. 将客体生物药物组装到 LDHs 层间可得到生物药物/ LDHs 纳米复合物, 纳米复合物的形成可降低药物的毒 副作用、提高其溶解度、稳定性并有效增强其靶向性等, 同时药物可与周围环境中阴离子进行缓慢离子交换, 从 而可实现药物的有效控释 ${ }^{[6-9]}$.

甲氨蝶呤(Methotrexatum, 简称 MTX) 是一类抗叶 酸类抗肿瘤药, 对多种恶性肿瘤具有良好的治疗作用, 主要通过对二氢叶酸还原酶的抑制而达到阻碍肿瘤细 胞的合成, 进而抑制肿瘤细胞的生长与繁殖 ${ }^{[10,11]}$. MTX 具有价格便宜、药效显著等优势, 但其毒副作用较大. 研究表明, 将甲氨蝶呤(MTX)组装到 LDH 层间后, 因静 电相互作用而使其稳定性大为增加; 相同药物浓度下, $\mathrm{MTX} / \mathrm{LDH}$ 复合物的药效约为药物本身的 5000 倍 ${ }^{[12]}$. Choy 等 ${ }^{[10,13]}$ 利用 LDH 为载体, 采用共沉淀法成功合成 了 MTX/LDH 纳米复合物, 并分别将 MTX和 MTX/LDH 作用于人类骨肉瘤细胞 (SaOS-2) 中, 研究发现 MTX/LDH 抑制肿瘤细胞生长的效率远远高于纯 MTX 的, 且体内释放半衰期延长, 器官靶向性增强.

由于生物药物/LDHs 纳米复合物尺寸小, 表面能高, 采用传统的共沉淀法制备时存在粒径分布难以控制、易 团聚、单分散性差等缺点 ${ }^{[14]}$, 对临床应用造成了一定的 影响. 我们课题组采用改进的共沉淀法 ${ }^{[15]}$ 成功制备出 了分散性好, 粒径分布均一的 MTX/LDH 复合物, 并初 步研究了复合物粒径对药物缓释效果的影响. 然而研究 表明阴离子在 LDH 层间的倾斜角度不同也会影响药物 释放速率 ${ }^{[16]}$, 因此当探索复合物粒径对药物缓释效果 影响时，必须保证合成复合物的阴离子在层间的倾斜角 度相同或相似的情况下来进行研究. 本文探索了保证复 合物阴离子在 LDH 层间的倾斜角度相似的情况下, 制 备出不同粒径纳米复合物的新途径, 并对这系列纳米复 合物的载药量和药物控释-缓释效果进行了系统的研究. 该研究为提高抗癌药物载体的载药量, 改善药物控释缓释效果提供了一条简单有效的新途径, 为 MTX/LDH 纳米复合物的临床应用打下了坚实的基础.

\section{2 结果与讨论}

\section{$2.1 \mathrm{XRD}$ 分析}

本文采用控制沉淀剂滴加速率的方法来制备系列
不同粒径的 MTX/LDH 纳米复合物, 即考察了一次性加 入、沉淀剂滴加速率分别为 $0.100 \mathrm{~mL} / \mathrm{s}, 0.050 \mathrm{~mL} / \mathrm{s}$ 和 $0.025 \mathrm{~mL} / \mathrm{s}$ 时制备的 MTX/LDH 纳米复合物的性质. 图 1 为沉淀剂不同滴加速率下制备的 MTX/LDH 纳米复合 物的 XRD 图, 从图中可以看出, 所有产品的 XRD 图谱 都表现出相似的特征衍射峰, 较好地保持了 LDH 的层 状结构 ${ }^{[13]}$; 与 $\mathrm{LDH}-\mathrm{NO}_{3}^{-}$(特征衍射峰 $d_{003}$ 对应的 $2 \theta$ 角为 $10.92^{\circ}$ )相比, 复合物的 $d_{003}$ 特征衍射峰均向低角度 方向偏移 ${ }^{[17]}$, 表明 MTX 成功插层到 LDH 层间, 使 LDH 的层间距增大. 随着滴加速率的减慢, 产物的衍射峰强 度先增加后减小, 后又有增强的趋势. 我们推测沉淀剂 加入速率过快 (一次性加入)时，体系中发生了快速成核 过程, 形成了大量晶核, 晶核来不及充分生长, 导致晶 粒结晶程度差, 具有较多缺陷, 这与其在 XRD 图中衍 射峰强度最低是一致的. 当沉淀剂的滴加速率为 0.100 $\mathrm{mL} / \mathrm{s}$-即滴加时间适中时, 形成的晶粒大小均匀, 结晶 程度最好, 故 XRD 图谱中其特征衍射峰强度最强. 加 料速率较慢 $(0.050 \mathrm{~mL} / \mathrm{s}$ 和 $0.025 \mathrm{~mL} / \mathrm{s})$ 时, 滴加时间相对 较长, 这样就造成了体系中晶粒成核和生长过程的同步 性降低，早成核的晶粒生长时间长，而晚成核的晶粒则 来不及长大, 导致粒子的多分散性增加, 使其结晶程度 相对降低. 从 XRD 图中可以看出, 当滴加速率为 0.100 $\mathrm{mL} / \mathrm{s}$ 时, 衍射峰变高且变窄, 表明在该条件下得到的 MTX/LDH 纳米复合物的结晶度较好, 粒子粒径较大.

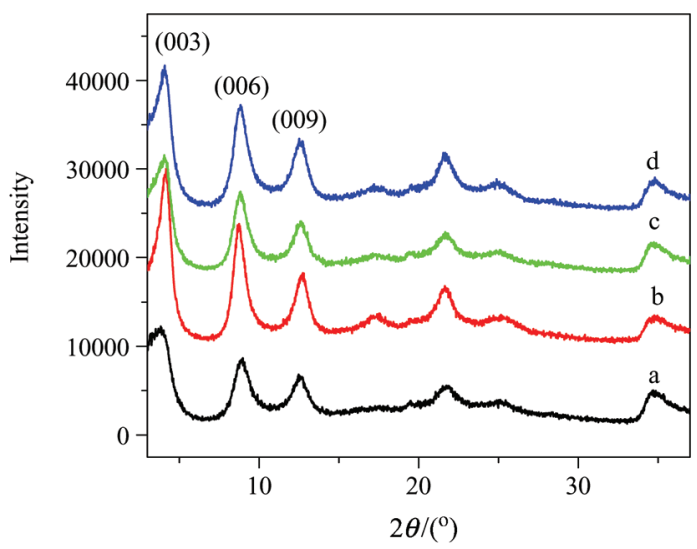

图 1 沉淀剂不同滴加速率下制得的 MTX/LDH 纳米复合物的 XRD 图

Figure 1 XRD patterns of MTX/LDH compounds obtained at different dropping speed of precipitator

(a) disposable dropping; (b) $0.100 \mathrm{~mL} / \mathrm{s}$; (c) $0.050 \mathrm{~mL} / \mathrm{s}$; (d) $0.025 \mathrm{~mL} / \mathrm{s}$

表 1 为沉淀剂不同滴加速率下得到的 MTX/LDH 纳 米复合物的 XRD 数据及其平均粒径. 根据布拉格公式, 可以计算出样品的层间距，由于 LDH 层板厚度为 0.48 $\mathrm{nm}^{[18]}$, 可以得到 $\mathrm{a} \sim \mathrm{d}$ 号样品的通道高度分别为 1.7993 , $1.6577,1.6693,1.6693 \mathrm{~nm}$. 甲氨蝶呤分子的长度为 2.12 $\mathrm{nm}^{[19]}$ ，据此我们模拟了不同滴速下制得的 MTX/LDH 纳米复合物空间排列示意图, 如图 2 所示: MTX 在 LDH 层间呈单分子倾斜排列, 倾斜的角度在 $51^{\circ}$ 到 $58^{\circ}$ 之间变 
化. 碱液一次性加入时, MTX 与层板间的夹角最大为 $58^{\circ}$. 有研究指出 ${ }^{[20]}$, 溶液 $\mathrm{pH}$ 值对插层阴离子在 $\mathrm{LDH}$ 层 间的倾斜角度有较大的影响, 随着溶液 $\mathrm{pH}$ 值的增加, 插层产物层间距随之增加, 进而导致客体分子在 LDH 层间倾斜角度的增大. 沉淀剂滴加速率的不同导致体系 中瞬间 $\mathrm{pH}$ 值的不同: 沉淀剂一次性加入时, 溶液 $\mathrm{pH}$ 值 变化剧烈, 瞬间达到较大值, 因此形成的 MTX/LDH 纳 米复合物的层间距最大, MTX 与层板间的夹角最大; 而 沉淀剂匀速缓慢加入时, 溶液中 $\mathrm{pH}$ 值变化缓慢, 因此 形成的 $\mathrm{MTX} / \mathrm{LDH}$ 纳米复合物的层间距几乎保持不变, MTX 与层板间的夹角也可以近似认为保持恒定. 据此 我们探索出一条保证复合物阴离子在层间的倾斜角度 相似的情况下, 制备出系列不同粒径纳米复合物的新途 径.

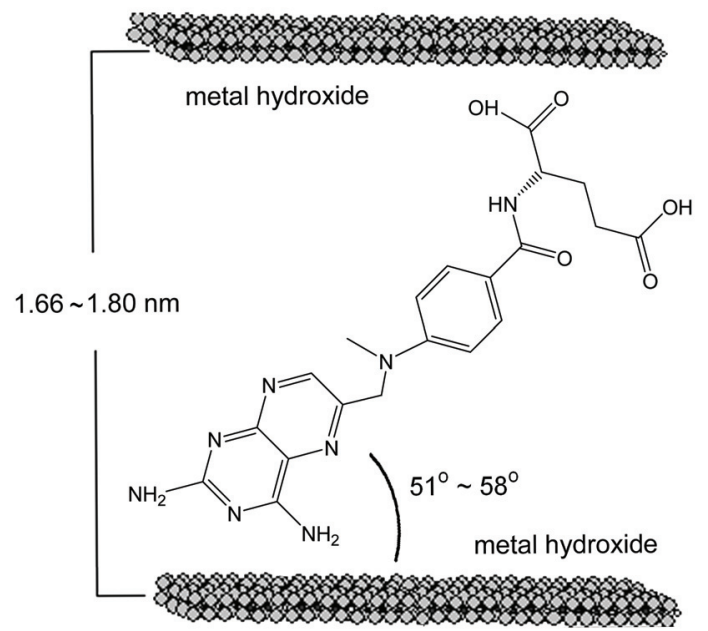

图 2 沉淀剂不同滴加速率下制得的 MTX/LDH 纳米复合物的排列方 式示意图

Figure 2 Scheme of the arrangement of MTX/LDH compounds obtained at different dropping speed of precipitator

\subsection{FT-IR 分析}

图 3 显示了沉淀剂不同滴加速率下制得的 $\mathrm{MTX} / \mathrm{LDH}$ 纳米复合物的 FT-IR 光谱图. 从图 3 可以看 出, $3400 \mathrm{~cm}^{-1}$ 处的宽峰对应于 LDH 层板上和层间水分 子的 $\mathrm{OH}^{-}$伸缩峰 ${ }^{[20]} .1386 \mathrm{~cm}^{-1}$ 处的 $\mathrm{NO}_{3}^{-}$吸收峰消失, 表明层间 MTX 取代了 $\mathrm{NO}_{3}^{-}$, 成功插入到 LDH 层间. 1614 和 $1456 \mathrm{~cm}^{-1}$ 处的吸收峰对应于 MTX 中芳香环的 $\mathrm{C}=\mathrm{C}$ 伸缩振动峰 ${ }^{[18]} .1207 \mathrm{~cm}^{-1}$ 处的吸收峰为 MTX 的

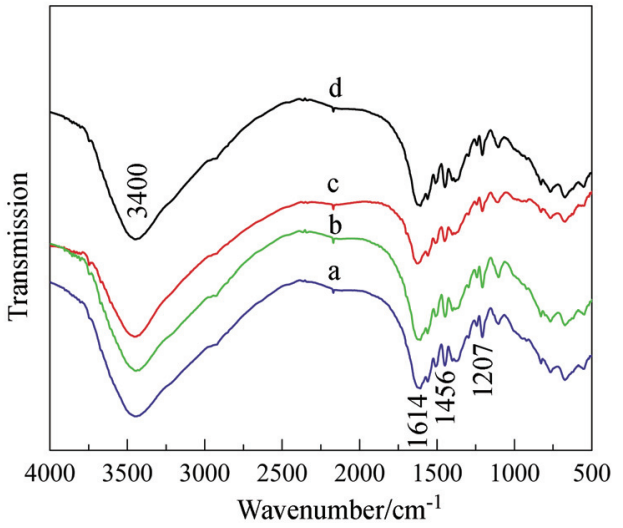

图 3 沉淀剂不同滴加速率下制得的 MTX/LDH 纳米复合物的 FT-IR 光谱

Figure 3 FT-IR spectra of MTX/LDH compounds obtained at different dropping speed of precipitator

(a) disposable dropping; (b) $0.100 \mathrm{~mL} / \mathrm{s}$; (c) $0.050 \mathrm{~mL} / \mathrm{s}$; (d) $0.025 \mathrm{~mL} / \mathrm{s}$

$\mathrm{C}-\mathrm{N}$ 键. 在低频振动区间, 出现的吸收峰对应着点阵 振动模式 ( $\mathrm{Al}-\mathrm{O}, \mathrm{Mg}-\mathrm{O}, \mathrm{Al}-\mathrm{O}-\mathrm{Mg}$ 等).

\subsection{TEM 分析}

沉淀剂不同滴加速率下得到的 MTX/LDH 纳米复合 物的形貌和粒径分布见图 4. 从图中可以看出不同滴加 速率下制备的 MTX/LDH 纳米复合物基本呈圆形，沉淀 剂滴加速率的不同导致了粒径的变化. 表 1 列出了不同 滴速下得到的纳米粒子平均粒径，平均粒径是通过测定 多张 $(>20) \mathrm{TEM}$ 图中粒子的粒径得到的. 从表 1 可以看 出，随着滴加速率减慢，粒子粒径先增大而后几乎保持 不变. 滴速为 $0.100 \mathrm{~mL} / \mathrm{s}$ 时得到的纳米粒子粒径最大, 这种变化规律也体现在 XRD 图上. 沉淀剂滴加速率过 快(一次性加入)时，导致体系中瞬间产生大量晶核，根 据质量守恒，成核量越大，最后形成的颗粒就越小，所 以沉淀剂一次性加入时得到的粒子粒径最小. 加料速率 减慢后 $(0.100$ 和 $0.050 \mathrm{~mL} / \mathrm{s})$, 先生成的微晶降低了体系 的成核能垒，溶液中的粒子以生长为主，得到的颗粒粒 径较大, 分散性好. 沉淀剂滴加速率过慢 $(0.025 \mathrm{~mL} / \mathrm{s})$ 时, 体系中晶粒成核和生长会发生同步性降低, 较早成核的 晶粒生长时间长，而晚成核的晶粒则来不及长大，导致 粒径的均匀性较差, 单分散性降低, 如图 4d. 0.100 和 $0.050 \mathrm{~mL} / \mathrm{s}$ 下制得的粒子的分散性较好且粒子形状均一 (图 $4 \mathrm{~b}$ 和 $4 \mathrm{c}$ ). 因此, 从理论上讲可以通过控制沉淀剂的 滴加速率来调控产物的粒径和单分散性.

表 1 沉淀剂不同滴加速率下制得的 MTX/LDH 的晶体结构数据

Table 1 Characteristic data of MTX/LDH compounds obtained at different dropping speed of precipitator

\begin{tabular}{cccccc}
\hline Sample & Dropping speed & Basal spacing $/ \mathrm{nm}$ & Interlayer spacing/nm & Angle of inclination $/\left({ }^{\circ}\right)$ & Mean particle size $/ \mathrm{nm}$ \\
\hline 1 & disposable dropping & 2.2793 & 1.7993 & 58.07 & 84.05 \\
2 & $0.100 \mathrm{~mL} / \mathrm{s}$ & 2.1377 & 1.6577 & 51.44 & 113.70 \\
3 & $0.050 \mathrm{~mL} / \mathrm{s}$ & 2.1493 & 1.6693 & 51.94 & 100.25 \\
4 & $0.025 \mathrm{~mL} / \mathrm{s}$ & 2.1493 & 1.6693 & 51.94 & 104.90 \\
\hline
\end{tabular}



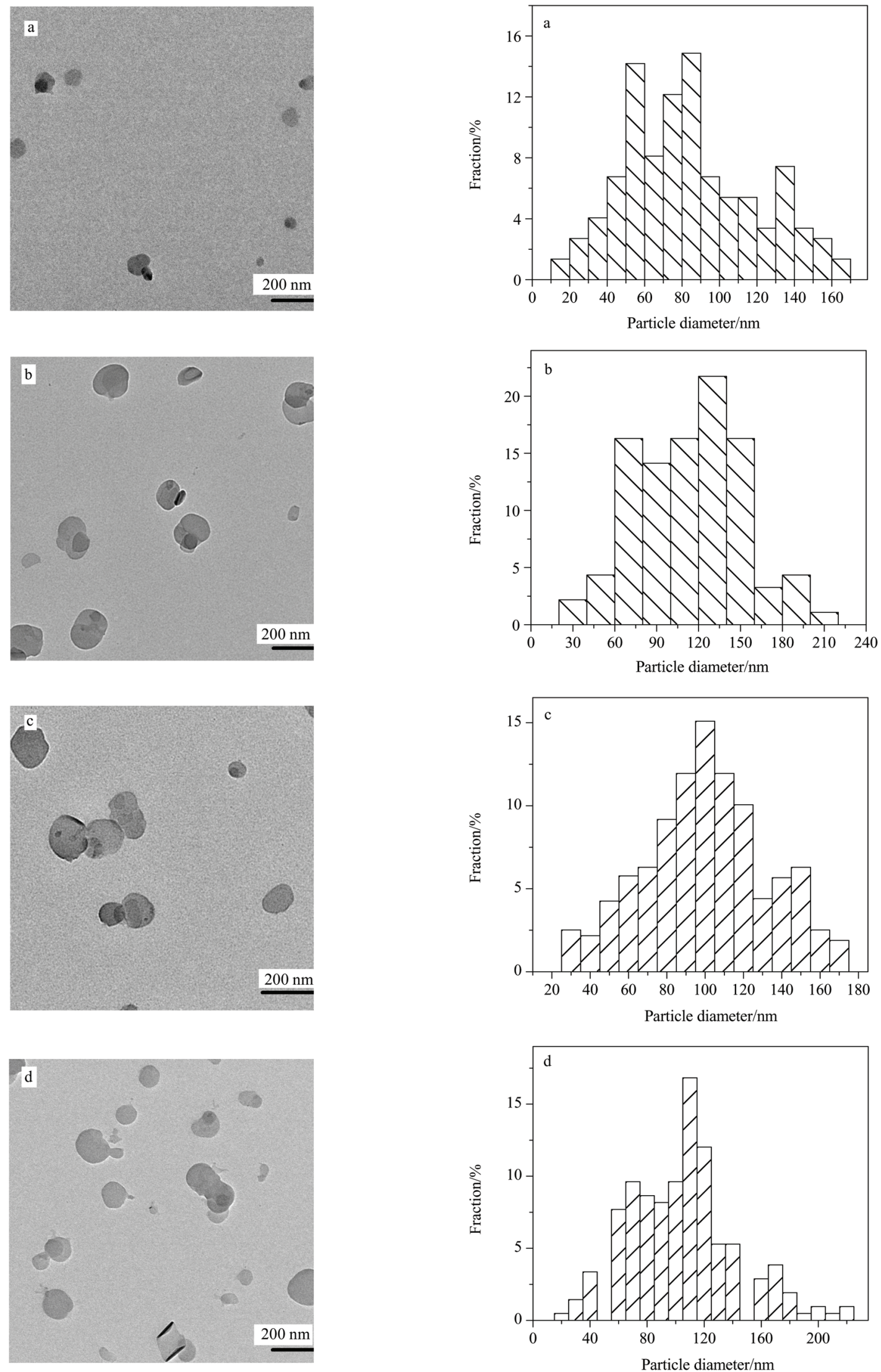

图 4 沉淀剂不同滴加速率下制得的 MTX/LDH 纳米复合物的 TEM 图和粒径分布图

Figure 4 TEM pictures and distribution of particle size of MTX/LDH compounds obtained at different dropping speed of precipitator $\mathrm{a}-$ disposable dropping; $\mathrm{b}-0.100 \mathrm{~mL} / \mathrm{s} ; \mathrm{c}-0.050 \mathrm{~mL} / \mathrm{s} ; \mathrm{d}-0.025 \mathrm{~mL} / \mathrm{s}$ 


\section{4 缓释性能研究}

通过载药量测定, 粒径为 $84.05,113.70,100.25$ 和 $104.90 \mathrm{~nm}$ 的 $\mathrm{MTX} / \mathrm{LDH}$ 复合物的载药量分别为 $52.3 \%$, $43.7 \%, 52.7 \%$ 和 $47.6 \%$. 从实验结果可以看出: 排除实 验误差的可能影响, 粒径小的纳米复合物的载药量较大. 图 5 为沉淀剂不同滴加速率下制得的 MTX/LDH 纳米复 合物的释放曲线图, 从图中可以看出 4 种样品均表现出 良好的缓释效果, 无突释现象, 但是其释放速率有所不 同.

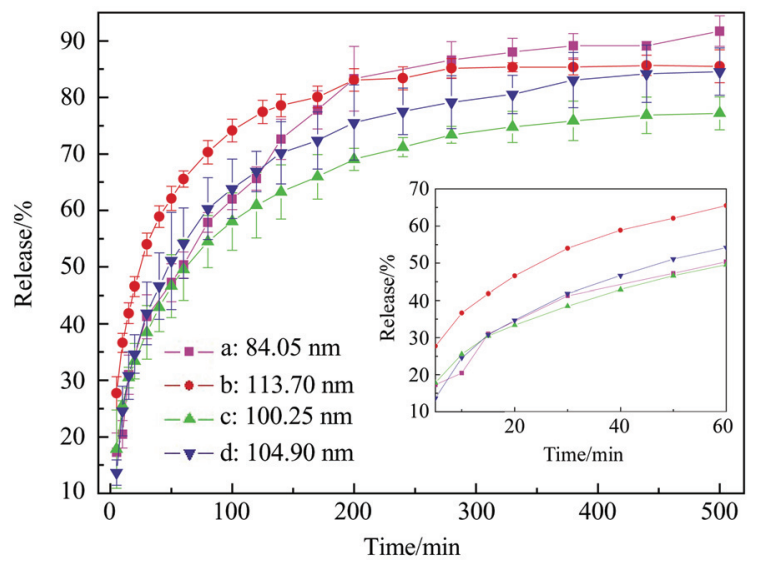

图 5 沉淀剂不同滴加速率下制得的 MTX/LDH 纳米复合物的缓释曲 线图

Figure 5 Release profiles of MTX/LDH compounds obtained at different dropping speed of precipitator

(a) disposable dropping; (b) $0.100 \mathrm{~mL} / \mathrm{s}$; (c) $0.050 \mathrm{~mL} / \mathrm{s}$; (d) $0.025 \mathrm{~mL} / \mathrm{s}$

理论上讲，阴离子在层间的倾斜角度会极大地影响 其释放速率 ${ }^{[16]}$, 分析 XRD 结果, 沉淀剂滴加速率为 $0.100,0.050$ 和 $0.025 \mathrm{~mL} / \mathrm{s}$ 时所得到的纳米粒子, MTX 阴 离子在层间的排列方式几乎相同，其倾斜角度分别为 $51.44^{\circ}, 51.94^{\circ}$ 和 $51.94^{\circ}$. 因此, 我们选择了这三个体系 以定性研究粒径对 MTX/LDH纳米复合物药物缓释效果 的影响, 以 $\mathrm{pH}=7.4$ 的磷酸缓冲液为溶剂, 控制温度为 $37{ }^{\circ} \mathrm{C}$, 所得缓释曲线见图 5. 由表 1 可知, 这三种样品 的平均粒径分别为 $113.70,100.25$ 和 $104.90 \mathrm{~nm}$. 粒径最 大的 $\mathrm{MTX} / \mathrm{LDH}$ 颗粒 $(113.70 \mathrm{~nm}$ )释放速率最快, $24 \mathrm{~min}$ 时释放速率已达到 50\%, 而粒径为 104.90 和 $100.25 \mathrm{~nm}$ 的 MTX/LDH 颗粒释放率达到 50\%时分别需要 48 和 63 min. $100 \mathrm{~min}$ 时, 粒径为 $113.70,104.90$ 和 $100.25 \mathrm{~nm}$ 的 $\mathrm{MTX} / \mathrm{LDH}$ 粒子的释放率分别达到 74\%, 64\%和 58\%. 从 以上结果分析可以看出, 粒径对 $\mathrm{MTX} / \mathrm{LDH}$ 纳米复合物 的药物释放速率有显著的影响, 粒径小的复合物释放速 率较慢, 其缓释效果优于粒径大的复合物, 这与我们之 前的报道是一致的 ${ }^{[15]}$.

沉淀剂一次性加入时得到的纳米复合物, MTX 阴 离子在 $\mathrm{LDH}$ 层间的倾斜角度为 $58.07^{\circ}$, 其排列方式与 另外三种样品的相差较大, 从图 5 我们可以定性看出, 其缓释曲线变化规律与其他三种样品的显著不同, 这表
明 MTX 在 LDH 层间的倾斜角度对其释放速率有影响. 段雪等 ${ }^{[16]}$ 也曾报道层间阴离子在层间的不同排布方式 会极大地影响其释放速率.

\section{5 释放动力学拟合}

一般来讲，生物药物/LDH 纳米复合物的释药机制 主要有两种: 离子交换机理和 $\mathrm{LDH}$ 片层的溶蚀 ${ }^{[21]}$. 为 了探索 MTX/LDH 纳米复合物的释药机理, 采用以下 4 种常用的数学模型对 $\mathrm{MTX} / \mathrm{LDH}$ 的释放过程进行拟 合 ${ }^{[22,23]}$.

(1) 一级方程: $\ln \left(1-M_{t} / M_{\infty}\right)=-k t$, 多用于描述释 放基质溶解所引发的药物释放行为和离子交换过程; (2) Higuchi 方程: $M_{t} / M_{\infty}=k t^{0.5}$, 它是以 Fickian 扩散定律为 基础提出的动力学方程, 适用于遵循扩散控释机理的释 药过程; (3) Bhaskar 方程: $\ln \left(1-M_{t} / M_{\infty}\right)=-k t^{0.65}$, 用于 描述不规则扩散或者粒内扩散机理; (4) Ritger-Peppas 方 程: $M_{t} / M_{\infty}=k t^{n}$, 其中 $n$ 为表征释放机制的特征参数. 当 $0.45<n<0.89$ 时, 药物的释放机制为 non-Fickian 扩散, 即扩散与溶蚀并存; 当 $n<0.45$ 时，为 Fickian 扩散; 当 $n>0.89$ 时, 为骨架溶蚀机制. 以上 4 个方程中, 其中 $M_{t}$ 为 $t$ 时间的累积释放量, $M_{\infty}$ 为 $\infty$ 时累积释放量, $M_{t} / M_{\infty}$ 为 $t$ 时累积释放率, $k$ 为释放常数. 拟合直线图见图 6 , 拟 合数据列于表 2 .

由拟合结果可知, 采用一级方程拟合得到的拟合因 子 $R$ 较小(仅样品 $\mathrm{a}$ 中 $R>0.96$, 样品 $\mathrm{b}, \mathrm{c}, \mathrm{d}$ 的 $R<0.95$ ), 拟合数据点离散严重, 说明 $\mathrm{MTX} / \mathrm{LDH}$ 纳米复合物的释 药机制不符合一级方程. 通过 Higuchi 方程拟合得到的 拟合因子 $R<0.96$, 说明 Higuchi 方程也不能很好地解释 $\mathrm{MTX} / \mathrm{LDH}$ 纳米复合物的释药机制. 而采用 Bhaskar 方 程拟合得到的拟合因子相对较高(仅样品 $\mathrm{b}$ 的 $R<0.96$ ), 初步判断 $\mathrm{MTX} / \mathrm{LDH}$ 纳米复合物在 $\mathrm{pH}=7.4$ 的磷酸缓冲 液中释放出 MTX 分子的机制属于扩散机制. 进一步采 用 Ritger-Peppas 方程，从拟合结果 $R>0.96$ 及 $n<0.45$ 可判定, $\mathrm{MTX} / \mathrm{LDH}$ 纳米复合物的释药机制属于离子交 换机理，且释放过程为扩散控制过程. 也就是说， $\mathrm{MTX} / \mathrm{LDH}$ 纳米复合物在释放过程中主要是缓释介质中 的磷酸氢根、磷酸二氢根离子将 LDH 片层中的 MTX 分 子置换出来，使得层间的 MTX 阴离子进入到缓释溶液 中, 因此. 当缓释介质中的磷酸氢根、磷酸二氢根阴离 子与层片边缘部位的 MTX 阴离子发生交换后, 逐渐形 成层板边缘部位层间距小、而中间部位层间距大的变形 结构，层间通道高度越大，其结构变形程度越大 ${ }^{[16]}$. 在 我们研究的范围内, 沉淀剂一次性加入时得到的 $\mathrm{MTX} / \mathrm{LDH}$ 纳米复合物的通道高度最大，因而其 MTX 阴离子经中间界面相区域扩散至层片边缘及至溶液相 时所受到的扩散阻力更大, 所经历的路程更长、更复杂, 即层间通道高度的增加导致交换反应中阴离子的扩散 速率降低，进而引起释放速率的降低，故沉淀剂一次性 加入时得到的样品其释放速率在开始的时间内最低. 

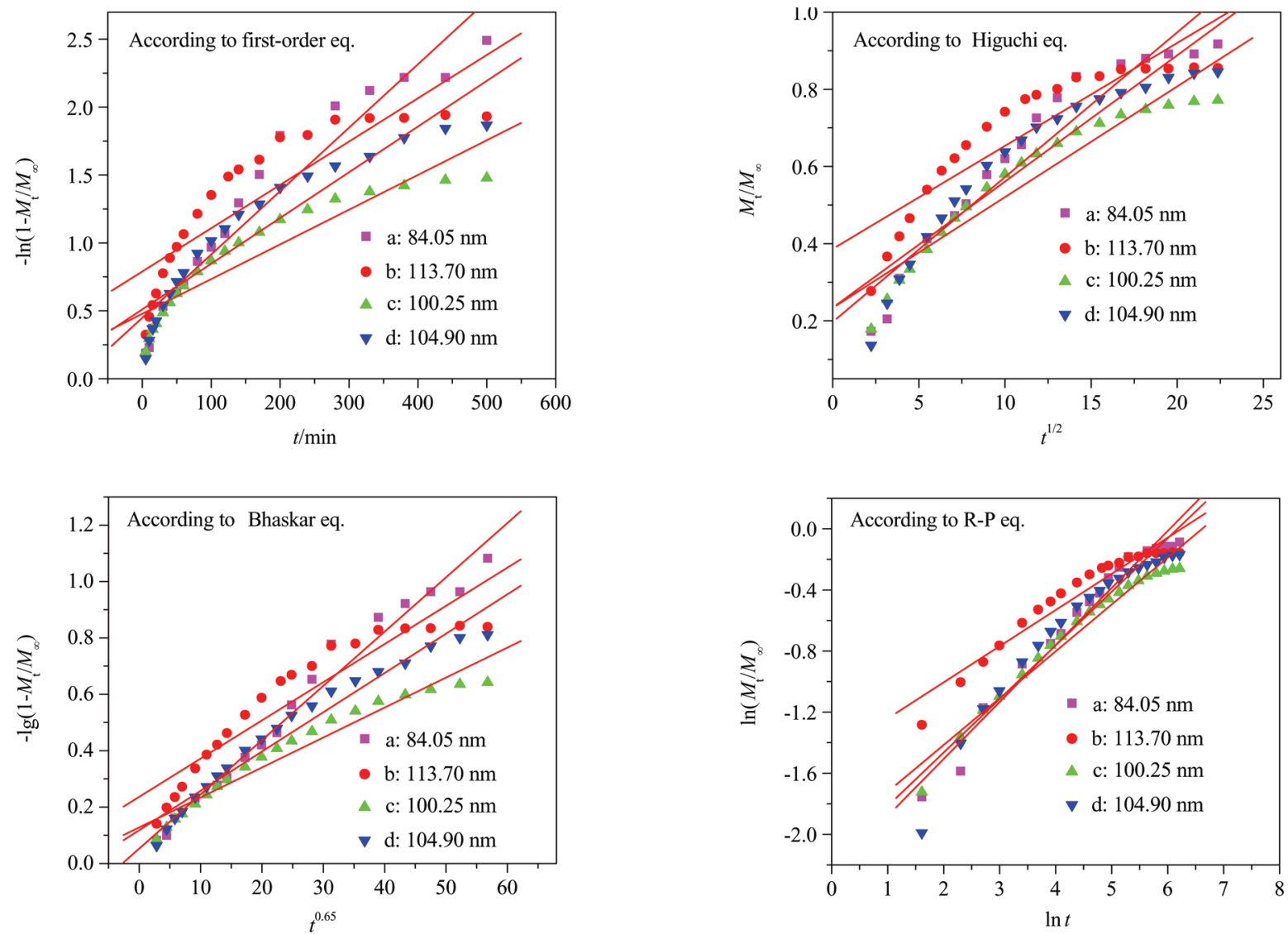

图 6 沉淀剂不同商加速率下制得的 $\mathrm{MTX} / \mathrm{LDH}$ 纳米复合物释放过程的动力学拟合直线

Figure 6 Plots of different kinetic models for the release of MTX from the MTX/LDH compounds obtained at different dropping speed of precipitator (a) disposable dropping; (b) $0.100 \mathrm{~mL} / \mathrm{s}$; (c) $0.050 \mathrm{~mL} / \mathrm{s}$; (d) $0.025 \mathrm{~mL} / \mathrm{s}$

表 2 沉淀剂不同滴加速率下制得的 MTX/LDH 纳米复合物释放过程的动力学拟合数据

Table 2 Fitting parameters of different kinetic models for the release of MTX from the MTX/LDH compounds obtained at different dropping speed of precipitator (a) disposable dropping; (b) $0.100 \mathrm{~mL} / \mathrm{s}$; (c) $0.050 \mathrm{~mL} / \mathrm{s}$; (d) $0.025 \mathrm{~mL} / \mathrm{s}$

\begin{tabular}{|c|c|c|c|c|c|c|c|c|c|}
\hline \multirow{2}{*}{ Sample } & \multicolumn{2}{|c|}{ first-order eq. } & \multicolumn{2}{|c|}{ Higuchi eq. } & \multicolumn{2}{|c|}{ Bhaskar eq. } & \multicolumn{3}{|c|}{ R-P eq. } \\
\hline & $R$ & $k_{1}$ & $R$ & $k_{\mathrm{H}}$ & $R$ & $k_{\mathrm{B}}$ & $R$ & $k$ & $n$ \\
\hline (a) & 0.9622 & 0.0047 & 0.9564 & 0.0375 & 0.9881 & 0.0192 & 0.9825 & 0.1051 & 0.3730 \\
\hline (b) & 0.8753 & 0.0032 & 0.9018 & 0.0267 & 0.9372 & 0.0135 & 0.9651 & 0.2279 & 0.2367 \\
\hline (c) & 0.9296 & 0.0026 & 0.9530 & 0.0287 & 0.9739 & 0.0106 & 0.9836 & 0.1315 & $0 . .3067$ \\
\hline (d) & 0.9384 & 0.0034 & 0.9405 & 0.0327 & 0.9771 & 0.0139 & 0.9633 & 0.1147 & 0.3507 \\
\hline
\end{tabular}

\section{3 结论}

本文采用控制沉淀剂滴加速率的方式制备了系列 不同粒径的 MTX/LDH 纳米复合物并在磷酸缓冲液中考 察了其缓释性能. 研究结果表明沉淀剂滴加速率对产物 的结晶度、粒径和层间排列方式都有影响，当沉淀剂滴 加速率为 $0.100 \mathrm{~mL} / \mathrm{s}$ 时, 得到的 MTX/LDH 纳米复合物 的结晶度最高, 粒径最大; 研究结果还表明小粒径的 $\mathrm{MTX} / \mathrm{LDH}$ 纳米复合物的载药量要高于大粒径的; 当 MTX 阴离子在层间的排列方式一致时, 粒径小的粒子 释放速率较慢, 缓释效果优于粒径大的粒子. 我们探索 出了一条保证复合物阴离子在层间的倾斜角度相似的 情况下, 制备不同粒径纳米复合物的新途径一一沉淀剂
匀速加入法.

\section{4 实验部分}

\section{1 共沉淀法合成 MTX/LDH}

称取 $0.7692 \mathrm{~g} \mathrm{Mg}\left(\mathrm{NO}_{3}\right)_{2} \bullet 6 \mathrm{H}_{2} \mathrm{O}, 0.5627 \mathrm{~g} \mathrm{Al}\left(\mathrm{NO}_{3}\right)_{3} \bullet$ $9 \mathrm{H}_{2} \mathrm{O}$ 溶于乙醇与水体积比为 $1: 3$ 的混合溶液中, 并转 移至三颈烧瓶中, 在恒定温度为 $60{ }^{\circ} \mathrm{C}$ 的条件下磁力摚 拌, 并通以氮气. 将 $0.3408 \mathrm{~g}$ 的 MTX 溶于 $20 \mathrm{~mL}$ 乙醇 与 $2 \mathrm{~mL} \mathrm{5 \%}$ 的氨水混合液中, 迅速倒入三颈烧瓶中, 用 $10 \%$ 的氨水作为沉淀剂, 控制沉淀剂以一定的滴加速率 滴加到上述混合溶液中, 调节溶液的 $\mathrm{pH}$ 值至 9.5 , 恒温 继续摚拌 $1 \mathrm{~h}$. 待反应结束后, 将所得产物用去离子水 
离心洗涤三遍, 并用无水乙醇洗涤两遍, 以清除吸附在 表面的 MTX. 将滤饼转移至反应釜中, $80{ }^{\circ} \mathrm{C}$ 条件下水 热处理 $24 \mathrm{~h}$. 水热处理完毕之后, 干燥得到产物.

\section{2 缓释性能研究}

根据测出的载药量计算出做缓释性能实验所需的 $\mathrm{MTX} / \mathrm{LDH}$ 的量, 将称量好的 MTX/LDH 纳米复合物置 于 $\mathrm{pH}$ 为 $7.4(500 \mathrm{~mL}$ ) 的磷酸缓冲液(按照中国药典 2005 年版第二部附录 XIVE 方法配置)中, 在 $(37 \pm 0.5){ }^{\circ} \mathrm{C}$ 下 恒温搅拌, 一定的时间间隔内, 取 $3 \mathrm{~mL}$ 溶液 10000 $\mathrm{r} / \mathrm{min}$ 下离心 $30 \mathrm{~s}$ 后取其上清液, 用紫外-可见分光光度 计在 $306 \mathrm{~nm}$ 处测其吸光度, 并根据标准曲线 $A=$ $0.050185 C+0.00062(r=0.99999, n=7)$ 计算 MTX 的累 积释放量.

本实验的仪器与试剂, 缓释性能标准曲线的绘制及 载药量的测定均列于 Supporting Information 中.

\section{References}

[1] Kwak, S. Y.; Jeong, Y. J.; Park, J. S. Solid State Ionics 2002, 151, 229.

[2] Zhang, H.; Qi, R.; Duan, X. Chin. J. Inorg. Chem. 2002, 18, 833. (张 慧，齐荣，段雪，无机化学学报, 2002, 18, 833.)

[3] Zhang, X.; Zhang, H.; Wei, M.; Evans, D. G.; Duan, X. Chem. J. Chin. Univ. 2004, 25, 1869. (张涃, 张慧, 卫敏, Evans, D. G., 段 雪, 高等学校化学学报, 2004, 25, 1869.)

[4] Nie, H.-Q.; Hou, W.-G. Acta Phys.-Chim. Sin. 2011, 27, 1783. (聂宏 寒, 侯万国, 物理化学学报, 2011, 27, 1783.)

[5] Jia, C.-X.; Zhang, X.-Q.; Li, S.-P. Chin. J. Chem, 2012, 30, 277.
[6] Choy, J. H.; Kwak, S. Y.; Jeong, Y. J.; Park, J. S. Angew. Chem. Int. Ed. 2000, 39, 4041 .

[7] Yang, J. H.; Han, Y. S.; Park, M.; Park, T.; Hwang, S. J.; Choy, J. H. Chem. Mater. 2007, 19, 2679.

[8] Katherine, M. T.; Scott, S. R.; Emmanuel, G. P. J. Controlled Release 2004, 95, 501.

[9] Kwak, S. Y.; Kriven, W. M.; Wallig, M. A.; Choy, J. H. Biomaterials 2004, 25, 5995.

[10] Oh, J. M.; Park, M.; Kim, S. T.; Jung, J. Y.; Kang, Y. G.; Choy, J. H. J. Phys. Chem. Solids 2006, 67, 1024.

[11] Tang, F.; Zheng, G.-H.; Yao, Q.-Z.; Lv, G.; Zhou, W.-F.; Wang, Q.-J. Acta Chim. Sinica 2006, 64, 249. (唐锋, 郑国海, 姚其正, 吕刚, 周卫芬, 王秋娟, 化学学报, 2006, 64, 249.)

[12] Xue, Y.-H.; Zhang, R.; Sun, X.-Y.; Wang, S.-L. Chin. J. Pharm. 2007，38，63. (薛燕华，张藸，孙晓宇，汪世龙，中国医药工业杂 志, 2007, 38, 63.)

[13] Oh, J. H.; Choi, S. J.; Kim, S. T.; Choy, J. H. Bioconjugate Chem. 2006, 17, 1411.

[14] Du, X.-Y.; Chen, Y.-Z.; Ma, Y.-X.; Li, F. Appl. Chem. Ind. 2008, 37, 1449. (杜雪岩，陈尹泽，马应霞，李芳，应用化工， 2008，37， 1449.)

[15] Wang, J.-Q.; Li, X.; Li, S.-P.; Zhong, H. Acta Chim. Sinica 2011, 69, 137. (王继芹，李金金，李淑萍，仲慧，化学学报, 2011, 69, 137.)

[16] Meng, J.-H.; Zhang, H.; Evans, D. G.; Duan, X. Chin. Sci. Bull. 2005, 50, 208. (孟锦宏，张慧，Evans，D. G.，段雪，科学通报， 2005, 50, 208.)

[17] Aisawa, S.; Ohnuma, Y.; Hirose, K.; Takahashi, S.; Hirahara, H.; Narita, E. Appl. Clay Sci. 2005, 28, 137.

[18] Aisawa, S.; Takahashi, S.; Ogasawara, W.; Umetsu, Y.; Narita, E. J. Solid State Chem. 2001, 162, 52.

[19] Choy, J. H.; Jung, J. S.; Oh, J. M.; Parka, M.; Jeong, J.; Kang, Y. K.; Han, O. J. Biomaterials 2004, 25, 3059.

[20] Li, B.-X.; He, J.; Evans, D. G.; Duan, X. Appl. Clay Sci. 2004, 27, 199.

[21] Xu, Z.-P.; Lu, G.-Q. Pure Appl. Chem. 2006, 78, 1771.

[22] Zhang, H.; Pan, D. K.; Duan, X. J. Phys. Chem. C 2009, 113, 12140.

[23] Li, F.-S.; Jin, L.; Han, J.-B.; Wei, M.; Li, C.-J. Ind. Eng. Chem. Res. 2009, 48,5590 . 